\title{
Supporting Information S3: An Evaluation Protocol for Subtype-Specific Breast Cancer Event Prediction
}

Herman MJ Sontrop ${ }^{1}$, Wim FJ Verhaegh ${ }^{1}$, Marcel JT Reinders ${ }^{2,4}$, Perry D Moerland ${ }^{3,4, *}$

1 Molecular Diagnostics Department, Philips Research, High Tech Campus 11, 5656 AE

Eindhoven, The Netherlands

2 Delft Bioinformatics Lab, Delft University of Technology, Mekelweg 4, 2628 CD Delft, The Netherlands

3 Bioinformatics Laboratory, Department of Clinical Epidemiology, Biostatistics, and Bioinformatics, Academic Medical Center, Meibergdreef 9, 1105 AZ Amsterdam, The Netherlands

4 Netherlands Bioinformatics Centre, Geert Grooteplein 28, 6525 GA Nijmegen, The Netherlands

* E-mail: p.d.moerland@amc.uva.nl

\section{Performance tables Nearest Centroid on balanced compendia}

Table S2. Typed (tp) vs. untyped (un) predictor performance.

\begin{tabular}{ccccccc}
\hline & & lumA & lumB & basal & Her2 & overall \\
\hline \multirow{4}{*}{ tp } & auc & 57.1 & 64.8 & 61.2 & 75.3 & 64.1 \\
& bar & 54.9 & 58.5 & 57.4 & 70.0 & 60.2 \\
& sen & 64.8 & 76.2 & 43.9 & 74.3 & 64.8 \\
& spc & 45.1 & 40.7 & 70.8 & 65.6 & 55.5 \\
& acc & 51.5 & 52.3 & 62.0 & 68.4 & 58.6 \\
& ppv & 36.6 & 38.6 & 42.4 & 51.3 & 41.5 \\
& npv & 72.3 & 78.0 & 72.2 & 84.0 & 76.5 \\
\hline & auc & 57.1 & 64.8 & 61.2 & 75.3 & 64.1 \\
& bar & 54.9 & 58.5 & 57.4 & 70.0 & 60.2 \\
& sen & 64.8 & 76.2 & 43.9 & 74.3 & 64.8 \\
up & spc & 45.1 & 40.7 & 70.8 & 65.6 & 55.5 \\
& acc & 51.5 & 52.3 & 62.0 & 68.4 & 58.6 \\
& ppv & 36.6 & 38.6 & 42.4 & 51.3 & 41.5 \\
npv & 72.3 & 78.0 & 72.2 & 84.0 & 76.5 \\
\hline
\end{tabular}

B.H.La.Lb 
Table S3. Typed (tp) vs. untyped (un) predictor performance.

\begin{tabular}{|c|c|c|c|c|c|c|}
\hline & & $\operatorname{lum} A$ & $\operatorname{lumB}$ & basal & Her2 & overall \\
\hline \multirow{7}{*}{ tp } & auc & 59.4 & 65.9 & 60.6 & 72.2 & 64.0 \\
\hline & bar & 56.4 & 58.5 & 56.7 & 67.5 & 59.8 \\
\hline & sen & 53.2 & 78.1 & 44.6 & 73.9 & 62.5 \\
\hline & $\mathrm{spc}$ & 59.7 & 39.0 & 68.8 & 61.0 & 57.1 \\
\hline & acc & 57.5 & 51.8 & 60.9 & 65.2 & 58.9 \\
\hline & $\mathrm{ppv}$ & 39.3 & 38.4 & 40.9 & 48.2 & 41.5 \\
\hline & $\mathrm{npv}$ & 72.4 & 78.7 & 72.0 & 82.7 & 75.8 \\
\hline \multirow{7}{*}{ up } & auc & 57.0 & 64.4 & 57.1 & 73.6 & 63.0 \\
\hline & bar & 55.0 & 58.9 & 54.7 & 68.8 & 59.3 \\
\hline & sen & 63.1 & 75.4 & 48.1 & 73.6 & 65.1 \\
\hline & $\mathrm{spc}$ & 46.9 & 42.3 & 61.3 & 63.9 & 53.6 \\
\hline & acc & 52.2 & 53.1 & 57.0 & 67.1 & 57.3 \\
\hline & $\mathrm{ppv}$ & 36.7 & 39.0 & 37.9 & 49.9 & 40.6 \\
\hline & npv & 72.2 & 78.1 & 70.9 & 83.3 & 75.9 \\
\hline
\end{tabular}

Table S4. Typed (tp) vs. untyped (un) predictor performance.

\begin{tabular}{ccccccc}
\hline & & lumA & lumB & basal & Her2 & overall \\
\hline \multirow{6}{*}{ tp } & $\mathbf{5 9 . 1}$ & $\mathbf{6 6 . 4}$ & 59.3 & $\mathbf{7 4 . 7}$ & $\mathbf{6 5 . 7}$ \\
& bar & $\mathbf{5 7 . 2}$ & $\mathbf{6 1 . 2}$ & 55.2 & $\mathbf{7 1 . 5}$ & $\mathbf{6 1 . 3}$ \\
& sen & 59.0 & 72.4 & 46.3 & $\mathbf{7 5 . 9}$ & 63.4 \\
& spc & $\mathbf{5 5 . 5}$ & $\mathbf{5 0 . 0}$ & 64.2 & $\mathbf{6 7 . 2}$ & $\mathbf{5 9 . 2}$ \\
& acc & $\mathbf{5 6 . 6}$ & $\mathbf{5 7 . 4}$ & 58.3 & $\mathbf{7 0 . 0}$ & $\mathbf{6 0 . 6}$ \\
& ppv & $\mathbf{3 9 . 7}$ & $\mathbf{4 1 . 6}$ & 38.9 & $\mathbf{5 2 . 9}$ & $\mathbf{4 3 . 1}$ \\
& npv & $\mathbf{7 3 . 5}$ & $\mathbf{7 9 . 1}$ & 71.3 & $\mathbf{8 5 . 1}$ & $\mathbf{7 6 . 9}$ \\
\hline & auc & 57.0 & 64.5 & $\mathbf{6 0 . 8}$ & 65.9 & 61.2 \\
& bar & 55.1 & 59.1 & $\mathbf{5 7 . 3}$ & 61.7 & 58.3 \\
& sen & $\mathbf{6 3 . 3}$ & $\mathbf{7 5 . 6}$ & 45.2 & 67.0 & 62.8 \\
up & spc & 46.8 & 42.6 & $\mathbf{6 9 . 5}$ & 56.5 & 53.8 \\
& acc & 52.2 & 53.4 & $\mathbf{6 1 . 6}$ & 59.9 & 56.8 \\
ppv & 36.8 & 39.1 & $\mathbf{4 2 . 1}$ & 43.1 & 39.8 \\
npv & 72.3 & 78.3 & $\mathbf{7 2 . 3}$ & 77.9 & 74.8 \\
\hline
\end{tabular}

$H \mid$ B.La.Lb 
Table S5. Typed (tp) vs. untyped (un) predictor performance.

\begin{tabular}{|c|c|c|c|c|c|c|}
\hline & & $\operatorname{lum} A$ & $\operatorname{lumB}$ & basal & Her2 & overall \\
\hline \multirow{7}{*}{ tp } & auc & 61.5 & 63.2 & 61.6 & 75.6 & 64.6 \\
\hline & bar & 56.3 & 57.5 & 57.4 & 70.8 & 60.5 \\
\hline & sen & 37.5 & 74.9 & 43.8 & 73.9 & 57.5 \\
\hline & $\mathrm{spc}$ & 75.1 & 40.1 & 70.9 & 67.7 & 63.4 \\
\hline & acc & 62.8 & 51.5 & 62.0 & 69.7 & 61.5 \\
\hline & ppv & 42.4 & 37.9 & 42.3 & 52.7 & 43.4 \\
\hline & $\mathrm{npv}$ & 71.2 & 76.8 & 72.2 & 84.2 & 75.4 \\
\hline \multirow{7}{*}{ up } & auc & 55.3 & 63.9 & 60.4 & 73.6 & 63.1 \\
\hline & bar & 53.8 & 58.5 & 57.1 & 68.5 & 59.5 \\
\hline & sen & 56.3 & 74.2 & 45.3 & 73.3 & 62.3 \\
\hline & $\mathrm{spc}$ & 51.3 & 42.8 & 69.0 & 63.7 & 56.7 \\
\hline & acc & 52.9 & 53.1 & 61.2 & 66.8 & 58.5 \\
\hline & $\mathrm{ppv}$ & 36.1 & 38.7 & 41.7 & 49.7 & 41.2 \\
\hline & $\mathrm{npv}$ & 70.7 & 77.5 & 72.2 & 83.1 & 75.6 \\
\hline
\end{tabular}

$L a \mid B . H . L b$

Table S6. Typed (tp) vs. untyped (un) predictor performance.

\begin{tabular}{|c|c|c|c|c|c|c|}
\hline & & $\operatorname{lum} A$ & $\operatorname{lumB}$ & basal & Her2 & overall \\
\hline \multirow{7}{*}{ tp } & auc & 55.5 & 65.0 & 60.4 & 74.3 & 63.1 \\
\hline & bar & 54.2 & 60.8 & 57.0 & 69.5 & 60.4 \\
\hline & sen & 66.1 & 71.7 & 43.7 & 73.4 & 63.7 \\
\hline & $\mathrm{spc}$ & 42.4 & 49.8 & 70.2 & 65.6 & 57.0 \\
\hline & acc & 50.1 & 57.0 & 61.6 & 68.2 & 59.2 \\
\hline & ppv & 35.9 & 41.2 & 41.7 & 51.0 & 41.9 \\
\hline & npv & 71.8 & 78.4 & 72.0 & 83.5 & 76.4 \\
\hline \multirow{7}{*}{ up } & auc & 57.0 & 60.6 & 60.8 & 74.1 & 63.0 \\
\hline & bar & 55.0 & 57.0 & 56.8 & 69.2 & 59.5 \\
\hline & sen & 63.2 & 66.4 & 44.3 & 74.6 & 62.1 \\
\hline & $\mathrm{spc}$ & 46.7 & 47.5 & 69.4 & 63.8 & 56.8 \\
\hline & acc & 52.1 & 53.7 & 61.2 & 67.3 & 58.6 \\
\hline & ppv & 36.7 & 38.1 & 41.3 & 50.2 & 41.2 \\
\hline & npv & 72.2 & 74.7 & 71.9 & 83.8 & 75.5 \\
\hline
\end{tabular}

Lb|B.H.La 
Table S7. Typed (tp) vs. untyped (un) predictor performance.

\begin{tabular}{|c|c|c|c|c|c|c|}
\hline & & $\operatorname{lum} A$ & $\operatorname{lumB}$ & basal & Her2 & overall \\
\hline \multirow{7}{*}{ tp } & auc & 65.6 & 68.2 & 60.5 & 74.4 & 65.7 \\
\hline & bar & 57.6 & 61.7 & 57.1 & 69.9 & 61.6 \\
\hline & sen & 32.1 & 78.9 & 44.3 & 72.6 & 57.0 \\
\hline & $\mathrm{spc}$ & 83.2 & 44.5 & 69.8 & 67.1 & 66.1 \\
\hline & acc & 66.5 & 55.7 & 61.5 & 68.9 & 63.1 \\
\hline & $\mathrm{ppv}$ & 48.6 & 41.0 & 41.7 & 51.8 & 45.0 \\
\hline & $\mathrm{npv}$ & 71.7 & 81.5 & 72.0 & 83.4 & 76.0 \\
\hline \multirow{7}{*}{ up } & auc & 56.5 & 63.9 & 59.8 & 71.4 & 62.7 \\
\hline & bar & 55.4 & 59.0 & 56.8 & 66.4 & 59.4 \\
\hline & sen & 61.3 & 72.9 & 47.7 & 72.2 & 63.5 \\
\hline & $\mathrm{spc}$ & 49.4 & 45.2 & 65.9 & 60.6 & 55.3 \\
\hline & acc & 53.3 & 54.2 & 59.9 & 64.4 & 58.0 \\
\hline & ppv & 37.2 & 39.4 & 40.6 & 47.3 & 40.9 \\
\hline & $\mathrm{npv}$ & 72.4 & 77.6 & 72.2 & 81.8 & 75.7 \\
\hline
\end{tabular}

B. $H \mid L a . L b$

Table S8. Typed (tp) vs. untyped (un) predictor performance.

\begin{tabular}{ccccccc}
\hline & & lumA & lumB & basal & Her2 & overall \\
\hline \multirow{4}{*}{ tp } & 55.7 & 62.5 & 57.0 & $\mathbf{7 4 . 1}$ & $\mathbf{6 2 . 9}$ \\
& bar & 54.9 & 57.1 & 54.0 & $\mathbf{7 0 . 6}$ & 59.1 \\
& sen & 63.9 & $\mathbf{7 6 . 3}$ & 42.6 & $\mathbf{7 3 . 8}$ & 64.2 \\
& spc & 45.8 & 37.8 & 65.3 & $\mathbf{6 7 . 3}$ & 54.1 \\
& acc & 51.7 & 50.4 & 57.9 & $\mathbf{6 9 . 4}$ & 57.4 \\
& ppv & 36.7 & 37.4 & 37.3 & $\mathbf{5 2 . 4}$ & 40.5 \\
npv & 72.2 & 76.8 & 70.2 & $\mathbf{8 4 . 1}$ & 75.7 \\
\hline & auc & 56.7 & 62.8 & $\mathbf{5 9 . 8}$ & 70.5 & 62.2 \\
bar & 55.3 & $\mathbf{5 8 . 5}$ & $\mathbf{5 6 . 2}$ & 65.9 & 59.0 \\
& sen & 61.4 & 72.0 & $\mathbf{4 6 . 7}$ & 71.1 & 62.8 \\
up & spc & $\mathbf{4 9 . 2}$ & $\mathbf{4 5 . 1}$ & $\mathbf{6 5 . 6}$ & 60.7 & $\mathbf{5 5 . 2}$ \\
& acc & $\mathbf{5 3 . 2}$ & $\mathbf{5 3 . 9}$ & $\mathbf{5 9 . 4}$ & 64.1 & 57.7 \\
ppv & 37.2 & $\mathbf{3 9 . 0}$ & $\mathbf{4 0 . 0}$ & 47.0 & 40.5 \\
npv & 72.5 & $\mathbf{7 6 . 9}$ & $\mathbf{7 1 . 8}$ & 81.3 & 75.3 \\
\hline
\end{tabular}

B.La|H.Lb 
Table S9. Typed (tp) vs. untyped (un) predictor performance.

\begin{tabular}{ccccccc}
\hline & & lumA & lumB & basal & Her2 & overall \\
\hline & auc & 56.8 & 64.0 & $\mathbf{6 1 . 6}$ & 72.2 & $\mathbf{6 3 . 7}$ \\
& bar & 54.8 & 59.4 & 56.3 & $\mathbf{6 8 . 7}$ & 59.8 \\
& sen & 55.6 & 67.7 & 43.3 & $\mathbf{7 4 . 8}$ & 60.3 \\
tp & spc & $\mathbf{5 3 . 9}$ & $\mathbf{5 1 . 2}$ & $\mathbf{6 9 . 4}$ & $\mathbf{6 2 . 7}$ & $\mathbf{5 9 . 3}$ \\
& acc & $\mathbf{5 4 . 5}$ & $\mathbf{5 6 . 6}$ & 60.8 & $\mathbf{6 6 . 6}$ & $\mathbf{5 9 . 6}$ \\
& ppv & 37.1 & $\mathbf{4 0 . 5}$ & 40.9 & $\mathbf{4 9 . 5}$ & $\mathbf{4 1 . 9}$ \\
& npv & 71.4 & 76.8 & 71.6 & $\mathbf{8 3 . 6}$ & 75.4 \\
\hline & auc & 56.4 & 63.2 & 59.5 & 71.2 & 62.3 \\
& bar & 54.8 & 59.0 & 56.9 & 66.6 & 59.3 \\
& sen & $\mathbf{6 0 . 1}$ & $\mathbf{7 2 . 7}$ & $\mathbf{4 7 . 8}$ & 72.2 & $\mathbf{6 3 . 2}$ \\
up & spc & 49.4 & 45.3 & 66.0 & 61.0 & 55.4 \\
& acc & 52.9 & 54.3 & 60.1 & 64.7 & 58.0 \\
& ppv & 36.8 & 39.4 & 40.8 & 47.6 & 40.9 \\
npv & 71.8 & 77.6 & 72.3 & 81.9 & 75.6 \\
\hline
\end{tabular}

B.Lb|H.La

Table S10. Typed (tp) vs. untyped (un) predictor performance.

\begin{tabular}{|c|c|c|c|c|c|c|}
\hline & & $\operatorname{lum} A$ & $\operatorname{lumB}$ & basal & Her2 & overall \\
\hline \multirow{7}{*}{ tp } & auc & 65.6 & 68.2 & 60.6 & 74.7 & 66.9 \\
\hline & bar & 57.6 & 61.7 & 56.7 & 71.5 & 61.9 \\
\hline & sen & 32.1 & 78.9 & 44.6 & 75.9 & 57.9 \\
\hline & $\mathrm{spc}$ & 83.2 & 44.5 & 68.8 & 67.2 & 65.9 \\
\hline & acc & 66.5 & 55.7 & 60.9 & 70.0 & 63.3 \\
\hline & $\mathrm{ppv}$ & 48.6 & 41.0 & 40.9 & 52.9 & 45.2 \\
\hline & npv & 71.7 & 81.5 & 72.0 & 85.1 & 76.3 \\
\hline \multirow{7}{*}{ up } & auc & 56.5 & 63.9 & 57.1 & 65.9 & 60.5 \\
\hline & bar & 55.4 & 59.0 & 54.7 & 61.7 & 57.7 \\
\hline & sen & 61.3 & 72.9 & 48.1 & 67.0 & 62.3 \\
\hline & $\mathrm{spc}$ & 49.4 & 45.2 & 61.3 & 56.5 & 53.1 \\
\hline & acc & 53.3 & 54.2 & 57.0 & 59.9 & 56.1 \\
\hline & $\mathrm{ppv}$ & 37.2 & 39.4 & 37.9 & 43.1 & 39.3 \\
\hline & npv & 72.4 & 77.6 & 70.9 & 77.9 & 74.4 \\
\hline
\end{tabular}

$B|H| L a . L b$ 
Table S11. Typed (tp) vs. untyped (un) predictor performance.

\begin{tabular}{ccccccc}
\hline & & lumA & lumB & basal & Her2 & overall \\
\hline & auc & $\mathbf{6 1 . 5}$ & 62.5 & $\mathbf{6 0 . 6}$ & $\mathbf{7 4 . 1}$ & $\mathbf{6 4 . 4}$ \\
& bar & $\mathbf{5 6 . 3}$ & 57.1 & $\mathbf{5 6 . 7}$ & $\mathbf{7 0 . 6}$ & $\mathbf{6 0 . 2}$ \\
& sen & 37.5 & $\mathbf{7 6 . 3}$ & 44.6 & $\mathbf{7 3 . 8}$ & 58.1 \\
tp & spc & $\mathbf{7 5 . 1}$ & 37.8 & $\mathbf{6 8 . 8}$ & $\mathbf{6 7 . 3}$ & $\mathbf{6 2 . 3}$ \\
& acc & $\mathbf{6 2 . 8}$ & 50.4 & $\mathbf{6 0 . 9}$ & $\mathbf{6 9 . 4}$ & $\mathbf{6 0 . 9}$ \\
& ppv & $\mathbf{4 2 . 4}$ & 37.4 & $\mathbf{4 0 . 9}$ & $\mathbf{5 2 . 4}$ & $\mathbf{4 2 . 8}$ \\
& npv & 71.2 & 76.8 & 72.0 & $\mathbf{8 4 . 1}$ & 75.3 \\
\hline \multirow{6}{*}{ up } & 55.3 & 62.8 & 57.1 & 70.5 & 61.4 \\
& bar & 53.8 & $\mathbf{5 8 . 5}$ & 54.7 & 65.9 & 58.2 \\
& sen & $\mathbf{5 6 . 3}$ & 72.0 & 48.1 & 71.1 & $\mathbf{6 1 . 9}$ \\
spc & 51.3 & $\mathbf{4 5 . 1}$ & 61.3 & 60.7 & 54.6 \\
& acc & 52.9 & $\mathbf{5 3 . 9}$ & 57.0 & 64.1 & 57.0 \\
& ppv & 36.1 & $\mathbf{3 9 . 0}$ & 37.9 & 47.0 & 39.9 \\
npv & 70.7 & $\mathbf{7 6 . 9}$ & 70.9 & 81.3 & 74.7 \\
\hline
\end{tabular}

Table S12. Typed (tp) vs. untyped (un) predictor performance.

\begin{tabular}{ccccccc}
\hline & & lumA & lumB & basal & Her2 & overall \\
\hline \multirow{6}{*}{ auc } & 56.8 & $\mathbf{6 5 . 0}$ & $\mathbf{6 0 . 6}$ & 72.2 & $\mathbf{6 3 . 6}$ \\
& bar & 54.8 & $\mathbf{6 0 . 8}$ & $\mathbf{5 6 . 7}$ & $\mathbf{6 8 . 7}$ & $\mathbf{6 0 . 2}$ \\
& sen & 55.6 & $\mathbf{7 1 . 7}$ & 44.6 & $\mathbf{7 4 . 8}$ & 61.7 \\
& spc & $\mathbf{5 3 . 9}$ & 49.8 & $\mathbf{6 8 . 8}$ & $\mathbf{6 2 . 7}$ & $\mathbf{5 8 . 8}$ \\
& acc & $\mathbf{5 4 . 5}$ & $\mathbf{5 7 . 0}$ & $\mathbf{6 0 . 9}$ & $\mathbf{6 6 . 6}$ & $\mathbf{5 9 . 7}$ \\
& ppv & 37.1 & $\mathbf{4 1 . 2}$ & $\mathbf{4 0 . 9}$ & $\mathbf{4 9 . 5}$ & 42.2 \\
npv & 71.4 & $\mathbf{7 8 . 4}$ & 72.0 & $\mathbf{8 3 . 6}$ & $\mathbf{7 5 . 9}$ \\
\hline & auc & 56.4 & 60.6 & 57.1 & 71.2 & 61.3 \\
& bar & 54.8 & 57.0 & 54.7 & 66.6 & 58.3 \\
& sen & $\mathbf{6 0 . 1}$ & 66.4 & 48.1 & 72.2 & 61.7 \\
up & spc & 49.4 & 47.5 & 61.3 & 61.0 & 54.8 \\
& acc & 52.9 & 53.7 & 57.0 & 64.7 & 57.1 \\
ppv & 36.8 & 38.1 & 37.9 & 47.6 & 39.9 \\
npv & 71.8 & 74.7 & 70.9 & 81.9 & 74.7 \\
\hline
\end{tabular}

$B|L b| H . L a$ 
Table S13. Typed (tp) vs. untyped (un) predictor performance.

\begin{tabular}{|c|c|c|c|c|c|c|}
\hline & & $\operatorname{lumA}$ & $\operatorname{lumB}$ & basal & Her2 & overall \\
\hline \multirow{7}{*}{ tp } & auc & 61.5 & 64.0 & 61.6 & 74.7 & 66.1 \\
\hline & bar & 56.3 & 59.4 & 56.3 & 71.5 & 60.9 \\
\hline & sen & 37.5 & 67.7 & 43.3 & 75.9 & 56.1 \\
\hline & $\mathrm{spc}$ & 75.1 & 51.2 & 69.4 & 67.2 & 65.7 \\
\hline & acc & 62.8 & 56.6 & 60.8 & 70.0 & 62.6 \\
\hline & $\mathrm{ppv}$ & 42.4 & 40.5 & 40.9 & 52.9 & 44.3 \\
\hline & npv & 71.2 & 76.8 & 71.6 & 85.1 & 75.5 \\
\hline \multirow{7}{*}{ up } & auc & 55.3 & 63.2 & 59.5 & 65.9 & 60.5 \\
\hline & bar & 53.8 & 59.0 & 56.9 & 61.7 & 57.9 \\
\hline & sen & 56.3 & 72.7 & 47.8 & 67.0 & 61.0 \\
\hline & $\mathrm{spc}$ & 51.3 & 45.3 & 66.0 & 56.5 & 54.8 \\
\hline & acc & 52.9 & 54.3 & 60.1 & 59.9 & 56.8 \\
\hline & $\mathrm{ppv}$ & 36.1 & 39.4 & 40.8 & 43.1 & 39.6 \\
\hline & npv & 70.7 & 777.6 & 72.3 & 77.9 & 74.3 \\
\hline
\end{tabular}

Table S14. Typed (tp) vs. untyped (un) predictor performance.

\begin{tabular}{ccccccc}
\hline & & lumA & lumB & basal & Her2 & overall \\
\hline \multirow{4}{*}{ auc } & 55.7 & $\mathbf{6 5 . 0}$ & 57.0 & $\mathbf{7 4 . 7}$ & $\mathbf{6 4 . 3}$ \\
& bar & 54.9 & $\mathbf{6 0 . 8}$ & 54.0 & $\mathbf{7 1 . 5}$ & $\mathbf{6 0 . 3}$ \\
& sen & 63.9 & $\mathbf{7 1 . 7}$ & 42.6 & $\mathbf{7 5 . 9}$ & $\mathbf{6 3 . 5}$ \\
& spc & 45.8 & 49.8 & 65.3 & $\mathbf{6 7 . 2}$ & $\mathbf{5 7 . 0}$ \\
& acc & 51.7 & $\mathbf{5 7 . 0}$ & 57.9 & $\mathbf{7 0 . 0}$ & $\mathbf{5 9 . 1}$ \\
& ppv & 36.7 & $\mathbf{4 1 . 2}$ & 37.3 & $\mathbf{5 2 . 9}$ & $\mathbf{4 1 . 8}$ \\
npv & 72.2 & $\mathbf{7 8 . 4}$ & 70.2 & $\mathbf{8 5 . 1}$ & $\mathbf{7 6 . 3}$ \\
\hline & auc & 56.7 & 60.6 & $\mathbf{5 9 . 8}$ & 65.9 & 60.3 \\
bar & 55.3 & 57.0 & $\mathbf{5 6 . 2}$ & 61.7 & 57.6 \\
& sen & 61.4 & 66.4 & $\mathbf{4 6 . 7}$ & 67.0 & 60.4 \\
up & 49.2 & 47.5 & $\mathbf{6 5 . 6}$ & 56.5 & 54.7 \\
& acc & $\mathbf{5 3 . 2}$ & 53.7 & $\mathbf{5 9 . 4}$ & 59.9 & 56.6 \\
ppv & 37.2 & 38.1 & $\mathbf{4 0 . 0}$ & 43.1 & 39.4 \\
npv & 72.5 & 74.7 & $\mathbf{7 1 . 8}$ & 77.9 & 74.0 \\
\hline
\end{tabular}

$H|L b| B . L a$ 
Table S15. Typed (tp) vs. untyped (un) predictor performance.

\begin{tabular}{|c|c|c|c|c|c|c|}
\hline & & $\operatorname{lum} A$ & $\operatorname{lumB}$ & basal & Her2 & overall \\
\hline \multirow{7}{*}{ tp } & auc & 61.5 & 65.0 & 60.5 & 74.4 & 64.8 \\
\hline & bar & 56.3 & 60.8 & 57.1 & 69.9 & 61.0 \\
\hline & sen & 37.5 & 71.7 & 44.3 & 72.6 & 56.5 \\
\hline & $\mathrm{spc}$ & 75.1 & 49.8 & 69.8 & 67.1 & 65.4 \\
\hline & acc & 62.8 & 57.0 & 61.5 & 68.9 & 62.5 \\
\hline & $\mathrm{ppv}$ & 42.4 & 41.2 & 41.7 & 51.8 & 44.3 \\
\hline & $\mathrm{npv}$ & 71.2 & 78.4 & 72.0 & 83.4 & 75.6 \\
\hline \multirow{7}{*}{ up } & auc & 55.3 & 60.6 & 59.8 & 71.4 & 61.8 \\
\hline & bar & 53.8 & 57.0 & 56.8 & 66.4 & 58.5 \\
\hline & sen & 56.3 & 66.4 & 47.7 & 72.2 & 60.7 \\
\hline & $\mathrm{spc}$ & 51.3 & 47.5 & 65.9 & 60.6 & 56.3 \\
\hline & acc & 52.9 & 53.7 & 59.9 & 64.4 & 57.7 \\
\hline & ppv & 36.1 & 38.1 & 40.6 & 47.3 & 40.4 \\
\hline & $\mathrm{npv}$ & 70.7 & 74.7 & 72.2 & 81.8 & 74.6 \\
\hline
\end{tabular}

$L a|L b| B . H$

Table S16. Typed (tp) vs. untyped (un) predictor performance.

\begin{tabular}{|c|c|c|c|c|c|c|}
\hline & & $\operatorname{lumA}$ & $\operatorname{lumB}$ & basal & Her2 & overall \\
\hline & auc & 61.5 & 65.0 & 60.6 & 74.7 & 66.1 \\
\hline & bar & 56.3 & 60.8 & 56.7 & 71.5 & 61.3 \\
\hline & sen & 37.5 & 71.7 & 44.6 & 75.9 & 57.4 \\
\hline tp & $\mathrm{spc}$ & 75.1 & 49.8 & 68.8 & 67.2 & 65.2 \\
\hline & acc & 62.8 & 57.0 & 60.9 & 70.0 & 62.7 \\
\hline & ppv & 42.4 & 41.2 & 40.9 & 52.9 & 44.5 \\
\hline & npv & 71.2 & 78.4 & 72.0 & 85.1 & 75.9 \\
\hline & auc & 55.3 & 60.6 & 57.1 & 65.9 & 59.4 \\
\hline & bar & 53.8 & 57.0 & 54.7 & 61.7 & 56.8 \\
\hline & sen & 56.3 & 66.4 & 48.1 & 67.0 & 59.5 \\
\hline up & $\mathrm{spc}$ & 51.3 & 47.5 & 61.3 & 56.5 & 54.1 \\
\hline & acc & 52.9 & 53.7 & 57.0 & 59.9 & 55.9 \\
\hline & ppv & 36.1 & 38.1 & 37.9 & 43.1 & 38.7 \\
\hline & npv & 70.7 & 74.7 & 70.9 & 77.9 & 73.3 \\
\hline
\end{tabular}

$B|H| L a \mid L b$ 


\section{Performance tables Nearest Centroid on unbalanced compendium}

Table S17. Typed (tp) vs. untyped (un) predictor performance.

\begin{tabular}{|c|c|c|c|c|c|c|}
\hline & & $\operatorname{lum} A$ & $\operatorname{lumB}$ & basal & Her2 & overall \\
\hline \multirow{7}{*}{ tp } & auc & 68.6 & 72.7 & 50.4 & 60.6 & 69.6 \\
\hline & bar & 51.8 & 63.2 & 49.5 & 58.1 & 65.1 \\
\hline & sen & 5.8 & 87.8 & 86.8 & 84.9 & 72.0 \\
\hline & $\mathrm{spc}$ & 97.9 & 38.6 & 12.2 & 31.3 & 58.2 \\
\hline & acc & 85.6 & 53.5 & 39.3 & 48.8 & 61.8 \\
\hline & ppv & 29.9 & 38.4 & 36.1 & 37.5 & 37.3 \\
\hline & npv & 87.1 & 87.9 & 62.1 & 80.9 & 85.8 \\
\hline \multirow{7}{*}{ up } & auc & 68.6 & 72.7 & 50.4 & 60.6 & 69.6 \\
\hline & bar & 51.8 & 63.2 & 49.5 & 58.1 & 65.1 \\
\hline & sen & 5.8 & 87.8 & 86.8 & 84.9 & 72.0 \\
\hline & $\mathrm{spc}$ & 97.9 & 38.6 & 12.2 & 31.3 & 58.2 \\
\hline & acc & 85.6 & 53.5 & 39.3 & 48.8 & 61.8 \\
\hline & ppv & 29.9 & 38.4 & 36.1 & 37.5 & 37.3 \\
\hline & npv & 87.1 & 87.9 & 62.1 & 80.9 & 85.8 \\
\hline
\end{tabular}

Table S18. Typed (tp) vs. untyped (un) predictor performance.

\begin{tabular}{ccccccc}
\hline & & lumA & lumB & basal & Her2 & overall \\
\hline \multirow{4}{*}{ auc } & 68.4 & 72.1 & $\mathbf{6 2 . 2}$ & 59.3 & 69.7 \\
& bar & 52.2 & 61.2 & $\mathbf{5 8 . 0}$ & 57.0 & 64.0 \\
tp & sen & 6.7 & $\mathbf{9 0 . 9}$ & 50.0 & 83.9 & 64.2 \\
& spc & $\mathbf{9 7 . 8}$ & 31.5 & $\mathbf{6 6 . 1}$ & 30.0 & $\mathbf{6 3 . 8}$ \\
& acc & $\mathbf{8 5 . 6}$ & 49.5 & $\mathbf{6 0 . 2}$ & 47.7 & $\mathbf{6 3 . 9}$ \\
& ppv & $\mathbf{3 2 . 9}$ & 36.6 & $\mathbf{4 5 . 6}$ & 36.9 & $\mathbf{3 8 . 0}$ \\
npv & 87.2 & $\mathbf{8 8 . 9}$ & $\mathbf{6 9 . 9}$ & 79.3 & 83.8 \\
\hline \multirow{6}{*}{ auc } & 68.0 & 72.2 & 50.4 & $\mathbf{6 1 . 3}$ & $\mathbf{7 0 . 0}$ \\
bar & 52.0 & $\mathbf{6 2 . 7}$ & 50.9 & $\mathbf{5 8 . 0}$ & $\mathbf{6 4 . 7}$ \\
up & sen & 6.9 & 86.1 & $\mathbf{8 1 . 7}$ & 83.2 & $\mathbf{7 0 . 0}$ \\
spc & 97.0 & $\mathbf{3 9 . 2}$ & 20.1 & $\mathbf{3 2 . 8}$ & 59.4 \\
& acc & 85.0 & $\mathbf{5 3 . 4}$ & 42.4 & $\mathbf{4 9 . 3}$ & 62.1 \\
ppv & 27.3 & $\mathbf{3 8 . 1}$ & 36.8 & $\mathbf{3 7 . 6}$ & 37.3 \\
npv & 87.1 & 86.7 & 65.6 & 80.0 & $\mathbf{8 5 . 2}$ \\
\hline
\end{tabular}

B|H.La.Lb 
Table S19. Typed (tp) vs. untyped (un) predictor performance.

\begin{tabular}{ccccccc}
\hline & & lumA & lumB & basal & Her2 & overall \\
\hline \multirow{6}{*}{ tp } & auc & 68.6 & 71.9 & 46.4 & $\mathbf{7 4 . 7}$ & $\mathbf{7 0 . 1}$ \\
& bar & 52.1 & $\mathbf{6 4 . 5}$ & 48.1 & $\mathbf{7 1 . 5}$ & $\mathbf{6 6 . 8}$ \\
& sen & 5.5 & $\mathbf{8 7 . 7}$ & 86.7 & 75.9 & $\mathbf{7 0 . 5}$ \\
& spc & $\mathbf{9 8 . 6}$ & $\mathbf{4 1 . 3}$ & 9.6 & $\mathbf{6 7 . 2}$ & $\mathbf{6 3 . 0}$ \\
& acc & $\mathbf{8 6 . 2}$ & $\mathbf{5 5 . 4}$ & 37.6 & $\mathbf{7 0 . 0}$ & $\mathbf{6 4 . 9}$ \\
& ppv & 40.2 & $\mathbf{3 9 . 4}$ & 35.3 & $\mathbf{5 2 . 9}$ & $\mathbf{3 9 . 7}$ \\
& npv & 87.2 & $\mathbf{8 8 . 6}$ & 55.8 & $\mathbf{8 5 . 1}$ & $\mathbf{8 6 . 1}$ \\
\hline \multirow{6}{*}{ upc } & 68.3 & $\mathbf{7 2 . 4}$ & $\mathbf{5 0 . 3}$ & 60.3 & 69.4 \\
& bar & 52.0 & 62.8 & $\mathbf{4 9 . 6}$ & 57.5 & 64.6 \\
& sen & $\mathbf{6 . 5}$ & 86.5 & 86.5 & 74.9 & 70.0 \\
spc & 97.5 & 39.1 & $\mathbf{1 2 . 6}$ & 40.2 & 59.3 \\
& acc & 85.4 & 53.5 & $\mathbf{3 9 . 5}$ & 51.5 & 62.0 \\
& ppv & 29.2 & 38.2 & $\mathbf{3 6 . 1}$ & 37.9 & 37.3 \\
npv & 87.1 & 86.9 & $\mathbf{6 2 . 3}$ & 76.7 & 85.1 \\
\hline
\end{tabular}

Table S20. Typed (tp) vs. untyped (un) predictor performance.

\begin{tabular}{ccccccc}
\hline & & lumA & lumB & basal & Her2 & overall \\
\hline \multirow{4}{*}{ auc } & $\mathbf{6 4 . 8}$ & 65.6 & $\mathbf{6 3 . 4}$ & $\mathbf{7 6 . 5}$ & 67.3 \\
& bar & $\mathbf{5 6 . 3}$ & 59.3 & $\mathbf{5 8 . 3}$ & $\mathbf{7 1 . 2}$ & 62.9 \\
& sen & $\mathbf{3 1 . 3}$ & 74.1 & 49.6 & 74.1 & 60.2 \\
& spc & 81.3 & $\mathbf{4 4 . 5}$ & $\mathbf{6 7 . 1}$ & $\mathbf{6 8 . 4}$ & $\mathbf{6 5 . 7}$ \\
& acc & 74.6 & 53.5 & $\mathbf{6 0 . 7}$ & $\mathbf{7 0 . 3}$ & $\mathbf{6 4 . 3}$ \\
& ppv & 20.5 & 36.8 & $\mathbf{4 6 . 2}$ & $\mathbf{5 3 . 3}$ & $\mathbf{3 7 . 7}$ \\
npv & $\mathbf{8 8 . 5}$ & 79.8 & $\mathbf{7 0 . 0}$ & $\mathbf{8 4 . 4}$ & 82.7 \\
\hline & auc & 63.0 & $\mathbf{7 2 . 1}$ & 49.7 & 61.2 & $\mathbf{6 8 . 8}$ \\
& bar & 54.6 & $\mathbf{6 2 . 7}$ & 49.6 & 58.4 & $\mathbf{6 4 . 3}$ \\
& sen & 19.9 & $\mathbf{8 5 . 8}$ & $\mathbf{8 5 . 6}$ & $\mathbf{8 3 . 0}$ & $\mathbf{7 3 . 2}$ \\
up & $\mathbf{8 9 . 2}$ & 39.6 & 13.6 & 33.8 & 55.5 \\
& acc & $\mathbf{8 0 . 0}$ & 53.6 & 39.8 & 49.9 & 60.0 \\
& ppv & $\mathbf{2 2 . 4}$ & $\mathbf{3 8 . 2}$ & 36.1 & 37.9 & 36.2 \\
npv & 87.9 & $\mathbf{8 6 . 5}$ & 62.5 & 80.4 & $\mathbf{8 5 . 7}$ \\
\hline
\end{tabular}

$L a \mid$ B.H.Lb 
Table S21. Typed (tp) vs. untyped (un) predictor performance.

\begin{tabular}{ccccccc}
\hline & & lumA & lumB & basal & Her2 & overall \\
\hline & auc & 65.9 & $\mathbf{7 1 . 9}$ & 46.7 & 56.3 & 67.5 \\
& bar & 51.5 & $\mathbf{6 4 . 7}$ & 48.6 & 57.2 & $\mathbf{6 4 . 9}$ \\
& sen & 4.8 & 74.6 & $\mathbf{8 9 . 5}$ & $\mathbf{8 4 . 2}$ & 67.0 \\
tp & spc & $\mathbf{9 8 . 2}$ & $\mathbf{5 4 . 7}$ & 7.8 & 30.3 & $\mathbf{6 2 . 8}$ \\
& acc & $\mathbf{8 5 . 7}$ & $\mathbf{6 0 . 7}$ & 37.4 & 47.9 & $\mathbf{6 3 . 9}$ \\
& ppv & $\mathbf{2 8 . 7}$ & $\mathbf{4 1 . 8}$ & 35.6 & 37.0 & 38.4 \\
& npv & 87.0 & 83.2 & 56.2 & 79.8 & 84.6 \\
\hline & auc & $\mathbf{6 7 . 1}$ & 70.2 & $\mathbf{5 0 . 1}$ & $\mathbf{6 1 . 2}$ & $\mathbf{6 8 . 9}$ \\
& bar & $\mathbf{5 2 . 3}$ & 62.3 & $\mathbf{4 9 . 8}$ & $\mathbf{5 8 . 2}$ & 64.4 \\
& sen & $\mathbf{8 . 6}$ & $\mathbf{8 2 . 7}$ & 85.6 & 82.3 & $\mathbf{6 9 . 8}$ \\
up & spc & 96.0 & 41.9 & $\mathbf{1 4 . 0}$ & $\mathbf{3 4 . 0}$ & 59.1 \\
& acc & 84.3 & 54.3 & $\mathbf{4 0 . 0}$ & $\mathbf{4 9 . 8}$ & 61.8 \\
& ppv & 25.1 & 38.3 & $\mathbf{3 6 . 2}$ & $\mathbf{3 7 . 8}$ & 37.1 \\
npv & $\mathbf{8 7 . 2}$ & $\mathbf{8 4 . 8}$ & $\mathbf{6 2 . 7}$ & 79.9 & $\mathbf{8 5 . 0}$ \\
\hline
\end{tabular}

$L b \mid$ B.H.La

Table S22. Typed (tp) vs. untyped (un) predictor performance.

\begin{tabular}{|c|c|c|c|c|c|c|}
\hline & & $\operatorname{lumA}$ & lumB & basal & Her2 & overall \\
\hline \multirow{7}{*}{ tp } & auc & 69.0 & 72.6 & 61.7 & 74.9 & 71.1 \\
\hline & bar & 52.5 & 63.1 & 57.7 & 70.4 & 65.9 \\
\hline & sen & 6.4 & 92.4 & 47.4 & 71.8 & 62.2 \\
\hline & $\mathrm{spc}$ & 98.6 & 33.7 & 68.0 & 69.0 & 69.5 \\
\hline & acc & 86.3 & 51.5 & 60.6 & 69.9 & 67.6 \\
\hline & ppv & 42.8 & 37.8 & 45.8 & 53.0 & 41.4 \\
\hline & npv & 87.3 & 91.1 & 69.4 & 83.4 & 84.2 \\
\hline \multirow{7}{*}{ up } & auc & 67.5 & 71.6 & 49.9 & 61.3 & 69.6 \\
\hline & bar & 52.2 & 62.4 & 50.3 & 58.2 & 64.5 \\
\hline & sen & 8.3 & 84.8 & 83.6 & 80.3 & 69.8 \\
\hline & $\mathrm{spc}$ & 96.1 & 40.0 & 16.9 & 36.0 & 59.2 \\
\hline & acc & 84.4 & 53.6 & 41.1 & 50.5 & 61.9 \\
\hline & ppv & 25.6 & 38.1 & 36.5 & 38.0 & 37.1 \\
\hline & npv & 87.2 & 85.8 & 64.2 & 78.9 & 85.0 \\
\hline
\end{tabular}

B.H $H a . L b$ 
Table S23. Typed (tp) vs. untyped (un) predictor performance.

\begin{tabular}{ccccccc}
\hline & & lumA & lumB & basal & Her2 & overall \\
\hline & auc & 64.9 & 65.2 & 45.6 & $\mathbf{7 3 . 6}$ & 68.1 \\
& bar & 53.0 & 59.3 & 48.8 & $\mathbf{7 0 . 3}$ & $\mathbf{6 5 . 0}$ \\
& sen & 8.3 & 75.8 & $\mathbf{9 5 . 4}$ & 74.9 & 68.1 \\
tp & spc & $\mathbf{9 7 . 7}$ & 42.9 & 2.1 & $\mathbf{6 5 . 7}$ & $\mathbf{6 1 . 9}$ \\
& acc & $\mathbf{8 5 . 8}$ & 52.8 & 36.0 & $\mathbf{6 8 . 7}$ & $\mathbf{6 3 . 5}$ \\
& ppv & $\mathbf{3 5 . 9}$ & 36.6 & 35.7 & $\mathbf{5 1 . 5}$ & $\mathbf{3 8 . 2}$ \\
& npv & 87.4 & 80.3 & 45.1 & $\mathbf{8 4 . 3}$ & 84.9 \\
\hline & auc & $\mathbf{6 6 . 2}$ & $\mathbf{7 1 . 2}$ & $\mathbf{5 0 . 1}$ & 62.0 & $\mathbf{6 9 . 3}$ \\
& bar & 52.7 & $\mathbf{6 2 . 7}$ & $\mathbf{4 9 . 8}$ & 59.1 & 64.6 \\
& sen & $\mathbf{1 1 . 0}$ & $\mathbf{8 4 . 5}$ & 84.9 & $\mathbf{8 2 . 6}$ & $\mathbf{7 0 . 8}$ \\
up & spc & 94.5 & 40.8 & $\mathbf{1 4 . 7}$ & 35.6 & 58.4 \\
& acc & 83.3 & $\mathbf{5 4 . 1}$ & $\mathbf{4 0 . 2}$ & 51.0 & 61.6 \\
& ppv & 23.9 & $\mathbf{3 8 . 4}$ & $\mathbf{3 6 . 2}$ & 38.5 & 37.0 \\
npv & 87.3 & $\mathbf{8 5 . 9}$ & $\mathbf{6 2 . 8}$ & 80.9 & $\mathbf{8 5 . 3}$ \\
\hline
\end{tabular}

B.La|H.Lb

Table S24. Typed (tp) vs. untyped (un) predictor performance.

\begin{tabular}{|c|c|c|c|c|c|c|}
\hline & & $\operatorname{lum} A$ & $\operatorname{lumB}$ & basal & Her2 & overall \\
\hline \multirow{7}{*}{ tp } & auc & 68.4 & 68.0 & 64.8 & 59.2 & 70.2 \\
\hline & bar & 54.5 & 63.4 & 60.8 & 54.3 & 64.8 \\
\hline & sen & 13.8 & 66.8 & 63.6 & 94.7 & 60.7 \\
\hline & $\mathrm{spc}$ & 95.3 & 59.9 & 57.9 & 13.8 & 69.0 \\
\hline & acc & 84.4 & 62.0 & 60.0 & 40.3 & 66.9 \\
\hline & $\mathrm{ppv}$ & 31.1 & 42.1 & 46.3 & 34.8 & 40.4 \\
\hline & $\mathrm{npv}$ & 87.8 & 80.6 & 73.7 & 84.6 & 83.6 \\
\hline \multirow{7}{*}{ up } & auc & 65.4 & 71.6 & 49.9 & 61.7 & 69.1 \\
\hline & bar & 53.1 & 62.6 & 49.8 & 58.3 & 64.4 \\
\hline & sen & 13.1 & 84.8 & 85.1 & 80.5 & 71.1 \\
\hline & $\mathrm{spc}$ & 93.2 & 40.4 & 14.4 & 36.1 & 57.7 \\
\hline & acc & 82.5 & 53.9 & 40.1 & 50.6 & 61.2 \\
\hline & $\mathrm{ppv}$ & 23.0 & 38.3 & 36.2 & 38.0 & 36.7 \\
\hline & $\mathrm{npv}$ & 87.5 & 86.0 & 62.8 & 79.2 & 85.2 \\
\hline
\end{tabular}

B.Lb|H.La 
Table S25. Typed (tp) vs. untyped (un) predictor performance.

\begin{tabular}{ccccccc}
\hline & & lumA & lumB & basal & Her2 & overall \\
\hline & auc & $\mathbf{6 9 . 0}$ & $\mathbf{7 2 . 6}$ & $\mathbf{6 2 . 2}$ & $\mathbf{7 4 . 7}$ & $\mathbf{7 1 . 8}$ \\
& bar & 52.5 & $\mathbf{6 3 . 1}$ & $\mathbf{5 8 . 0}$ & $\mathbf{7 1 . 5}$ & $\mathbf{6 6 . 3}$ \\
& sen & 6.4 & $\mathbf{9 2 . 4}$ & 50.0 & 75.9 & 63.5 \\
tp & spc & $\mathbf{9 8 . 6}$ & 33.7 & $\mathbf{6 6 . 1}$ & $\mathbf{6 7 . 2}$ & $\mathbf{6 9 . 0}$ \\
& acc & $\mathbf{8 6 . 3}$ & 51.5 & $\mathbf{6 0 . 2}$ & $\mathbf{7 0 . 0}$ & $\mathbf{6 7 . 6}$ \\
& ppv & $\mathbf{4 2 . 8}$ & 37.8 & $\mathbf{4 5 . 6}$ & $\mathbf{5 2 . 9}$ & $\mathbf{4 1 . 5}$ \\
npv & 87.3 & $\mathbf{9 1 . 1}$ & $\mathbf{6 9 . 9}$ & $\mathbf{8 5 . 1}$ & 84.5 \\
\hline \multirow{6}{*}{ auc } & 67.5 & 71.6 & 50.4 & 60.3 & 69.6 \\
& bar & 52.2 & 62.4 & 50.9 & 57.5 & 64.3 \\
& sen & $\mathbf{8 . 3}$ & 84.8 & $\mathbf{8 1 . 7}$ & 74.9 & $\mathbf{6 8 . 4}$ \\
spc & 96.1 & $\mathbf{4 0 . 0}$ & 20.1 & 40.2 & 60.1 \\
& acc & 84.4 & $\mathbf{5 3 . 6}$ & 42.4 & 51.5 & 62.3 \\
ppv & 25.6 & $\mathbf{3 8 . 1}$ & 36.8 & 37.9 & 37.2 \\
npv & 87.2 & 85.8 & 65.6 & 76.7 & 84.7 \\
\hline
\end{tabular}

Table S26. Typed (tp) vs. untyped (un) predictor performance.

\begin{tabular}{ccccccc}
\hline & & lumA & lumB & basal & Her2 & overall \\
\hline \multirow{4}{*}{ auc } & $\mathbf{6 4 . 8}$ & 65.2 & $\mathbf{6 2 . 2}$ & $\mathbf{7 3 . 6}$ & 68.0 \\
& bar & $\mathbf{5 6 . 3}$ & 59.3 & $\mathbf{5 8 . 0}$ & $\mathbf{7 0 . 3}$ & 62.9 \\
& sen & $\mathbf{3 1 . 3}$ & 75.8 & 50.0 & 74.9 & 61.1 \\
& spc & 81.3 & $\mathbf{4 2 . 9}$ & $\mathbf{6 6 . 1}$ & $\mathbf{6 5 . 7}$ & $\mathbf{6 4 . 7}$ \\
& acc & 74.6 & 52.8 & $\mathbf{6 0 . 2}$ & $\mathbf{6 8 . 7}$ & $\mathbf{6 3 . 8}$ \\
& ppv & 20.5 & 36.6 & $\mathbf{4 5 . 6}$ & $\mathbf{5 1 . 5}$ & $\mathbf{3 7 . 4}$ \\
npv & $\mathbf{8 8 . 5}$ & 80.3 & $\mathbf{6 9 . 9}$ & $\mathbf{8 4 . 3}$ & 82.8 \\
\hline & auc & 63.0 & $\mathbf{7 1 . 2}$ & 50.4 & 62.0 & $\mathbf{6 9 . 0}$ \\
& bar & 54.6 & $\mathbf{6 2 . 7}$ & 50.9 & 59.1 & $\mathbf{6 4 . 4}$ \\
& sen & 19.9 & $\mathbf{8 4 . 5}$ & $\mathbf{8 1 . 7}$ & $\mathbf{8 2 . 6}$ & $\mathbf{7 1 . 7}$ \\
up & $\mathbf{8 9 . 2}$ & 40.8 & 20.1 & 35.6 & 57.1 \\
& acc & $\mathbf{8 0 . 0}$ & $\mathbf{5 4 . 1}$ & 42.4 & 51.0 & 60.8 \\
& ppv & $\mathbf{2 2 . 4}$ & $\mathbf{3 8 . 4}$ & 36.8 & 38.5 & 36.6 \\
npv & 87.9 & $\mathbf{8 5 . 9}$ & 65.6 & 80.9 & $\mathbf{8 5 . 4}$ \\
\hline
\end{tabular}

$B|L a| H . L b$ 
Table S27. Typed (tp) vs. untyped (un) predictor performance.

\begin{tabular}{|c|c|c|c|c|c|c|}
\hline & & $\operatorname{lum} A$ & lumB & basal & Her2 & overall \\
\hline \multirow{7}{*}{ tp } & auc & 68.4 & 71.9 & 62.2 & 59.2 & 69.4 \\
\hline & bar & 54.5 & 64.7 & 58.0 & 54.3 & 64.5 \\
\hline & sen & 13.8 & 74.6 & 50.0 & 94.7 & 60.5 \\
\hline & $\mathrm{spc}$ & 95.3 & 54.7 & 66.1 & 13.8 & 68.5 \\
\hline & acc & 84.4 & 60.7 & 60.2 & 40.3 & 66.5 \\
\hline & $\mathrm{ppv}$ & 31.1 & 41.8 & 45.6 & 34.8 & 39.9 \\
\hline & $\mathrm{npv}$ & 87.8 & 83.2 & 69.9 & 84.6 & 83.4 \\
\hline \multirow{7}{*}{ up } & auc & 65.4 & 70.2 & 50.4 & 61.7 & 69.0 \\
\hline & bar & 53.1 & 62.3 & 50.9 & 58.3 & 64.2 \\
\hline & sen & 13.1 & 82.7 & 81.7 & 80.5 & 69.4 \\
\hline & $\mathrm{spc}$ & 93.2 & 41.9 & 20.1 & 36.1 & 59.1 \\
\hline & acc & 82.5 & 54.3 & 42.4 & 50.6 & 61.7 \\
\hline & ppv & 23.0 & 38.3 & 36.8 & 38.0 & 36.9 \\
\hline & npv & 87.5 & 84.8 & 65.6 & 79.2 & 84.8 \\
\hline
\end{tabular}

Table S28. Typed (tp) vs. untyped (un) predictor performance.

\begin{tabular}{ccccccc}
\hline & & lumA & lumB & basal & Her2 & overall \\
\hline \multirow{6}{*}{ auc } & $\mathbf{6 4 . 8}$ & 68.0 & $\mathbf{6 4 . 8}$ & $\mathbf{7 4 . 7}$ & $\mathbf{7 0 . 4}$ \\
& bar & $\mathbf{5 6 . 3}$ & $\mathbf{6 3 . 4}$ & $\mathbf{6 0 . 8}$ & $\mathbf{7 1 . 5}$ & $\mathbf{6 5 . 1}$ \\
& sen & $\mathbf{3 1 . 3}$ & 66.8 & 63.6 & 75.9 & 60.9 \\
& spc & 81.3 & $\mathbf{5 9 . 9}$ & $\mathbf{5 7 . 9}$ & $\mathbf{6 7 . 2}$ & $\mathbf{6 9 . 2}$ \\
& acc & 74.6 & $\mathbf{6 2 . 0}$ & $\mathbf{6 0 . 0}$ & $\mathbf{7 0 . 0}$ & $\mathbf{6 7 . 1}$ \\
& ppv & 20.5 & $\mathbf{4 2 . 1}$ & $\mathbf{4 6 . 3}$ & $\mathbf{5 2 . 9}$ & $\mathbf{4 0 . 6}$ \\
npv & $\mathbf{8 8 . 5}$ & 80.6 & $\mathbf{7 3 . 7}$ & $\mathbf{8 5 . 1}$ & 83.7 \\
\hline & auc & 63.0 & $\mathbf{7 1 . 6}$ & 49.9 & 60.3 & 68.4 \\
& bar & 54.6 & 62.6 & 49.8 & 57.5 & 64.0 \\
& sen & 19.9 & $\mathbf{8 4 . 8}$ & $\mathbf{8 5 . 1}$ & 74.9 & $\mathbf{7 1 . 4}$ \\
up & spc & $\mathbf{8 9 . 2}$ & 40.4 & 14.4 & 40.2 & 56.6 \\
& acc & $\mathbf{8 0 . 0}$ & 53.9 & 40.1 & 51.5 & 60.4 \\
ppv & $\mathbf{2 2 . 4}$ & 38.3 & 36.2 & 37.9 & 36.2 \\
npv & 87.9 & $\mathbf{8 6 . 0}$ & 62.8 & 76.7 & $\mathbf{8 5 . 1}$ \\
\hline
\end{tabular}

$H|L a| B . L b$ 
Table S29. Typed (tp) vs. untyped (un) predictor performance.

\begin{tabular}{|c|c|c|c|c|c|c|}
\hline & & $\operatorname{lum} A$ & lumB & basal & Her2 & overall \\
\hline \multirow{7}{*}{ tp } & auc & 64.9 & 71.9 & 45.6 & 74.7 & 69.7 \\
\hline & bar & 53.0 & 64.7 & 48.8 & 71.5 & 66.9 \\
\hline & sen & 8.3 & 74.6 & 95.4 & 75.9 & 67.8 \\
\hline & $\mathrm{spc}$ & 97.7 & 54.7 & 2.1 & 67.2 & 65.9 \\
\hline & acc & 85.8 & 60.7 & 36.0 & 70.0 & 66.4 \\
\hline & $\mathrm{ppv}$ & 35.9 & 41.8 & 35.7 & 52.9 & 40.7 \\
\hline & $\mathrm{npv}$ & 87.4 & 83.2 & 45.1 & 85.1 & 85.6 \\
\hline \multirow{7}{*}{ up } & auc & 66.2 & 70.2 & 50.1 & 60.3 & 68.7 \\
\hline & bar & 52.7 & 62.3 & 49.8 & 57.5 & 64.1 \\
\hline & sen & 11.0 & 82.7 & 84.9 & 74.9 & 68.9 \\
\hline & $\mathrm{spc}$ & 94.5 & 41.9 & 14.7 & 40.2 & 59.2 \\
\hline & acc & 83.3 & 54.3 & 40.2 & 51.5 & 61.7 \\
\hline & $\mathrm{ppv}$ & 23.9 & 38.3 & 36.2 & 37.9 & 36.9 \\
\hline & npv & 87.3 & 84.8 & 62.8 & 76.7 & 84.6 \\
\hline
\end{tabular}

Table S30. Typed (tp) vs. untyped (un) predictor performance.

\begin{tabular}{|c|c|c|c|c|c|c|}
\hline & & $\operatorname{lumA}$ & lumB & basal & Her2 & overall \\
\hline \multirow{7}{*}{ tp } & auc & 64.8 & 71.9 & 61.7 & 74.9 & 68.2 \\
\hline & bar & 56.3 & 64.7 & 57.7 & 70.4 & 64.4 \\
\hline & sen & 31.3 & 74.6 & 47.4 & 71.8 & 59.5 \\
\hline & $\mathrm{spc}$ & 81.3 & 54.7 & 68.0 & 69.0 & 69.2 \\
\hline & acc & 74.6 & 60.7 & 60.6 & 69.9 & 66.7 \\
\hline & ppv & 20.5 & 41.8 & 45.8 & 53.0 & 40.1 \\
\hline & npv & 88.5 & 83.2 & 69.4 & 83.4 & 83.2 \\
\hline \multirow{7}{*}{ up } & auc & 63.0 & 70.2 & 49.9 & 61.3 & 68.4 \\
\hline & bar & 54.6 & 62.3 & 50.3 & 58.2 & 64.0 \\
\hline & sen & 19.9 & 82.7 & 83.6 & 80.3 & 71.0 \\
\hline & $\mathrm{spc}$ & 89.2 & 41.9 & 16.9 & 36.0 & 57.0 \\
\hline & acc & 80.0 & 54.3 & 41.1 & 50.5 & 60.6 \\
\hline & ppv & 22.4 & 38.3 & 36.5 & 38.0 & 36.3 \\
\hline & npv & 87.9 & 84.8 & 64.2 & 78.9 & 85.1 \\
\hline
\end{tabular}


Table S31. Typed (tp) vs. untyped (un) predictor performance.

\begin{tabular}{|c|c|c|c|c|c|c|}
\hline & & $\operatorname{lumA}$ & $\operatorname{lumB}$ & basal & Her2 & overall \\
\hline \multirow{7}{*}{ tp } & auc & 64.8 & 71.9 & 62.2 & 74.7 & 69.9 \\
\hline & bar & 56.3 & 64.7 & 58.0 & 71.5 & 64.8 \\
\hline & sen & 31.3 & 74.6 & 50.0 & 75.9 & 60.8 \\
\hline & $\mathrm{spc}$ & 81.3 & 54.7 & 66.1 & 67.2 & 68.8 \\
\hline & acc & 74.6 & 60.7 & 60.2 & 70.0 & 66.7 \\
\hline & $\mathrm{ppv}$ & 20.5 & 41.8 & 45.6 & 52.9 & 40.2 \\
\hline & $\mathrm{npv}$ & 88.5 & 83.2 & 69.9 & 85.1 & 83.5 \\
\hline \multirow{7}{*}{ up } & auc & 63.0 & 70.2 & 50.4 & 60.3 & 68.3 \\
\hline & bar & 54.6 & 62.3 & 50.9 & 57.5 & 63.8 \\
\hline & sen & 19.9 & 82.7 & 81.7 & 74.9 & 69.7 \\
\hline & $\mathrm{spc}$ & 89.2 & 41.9 & 20.1 & 40.2 & 57.9 \\
\hline & acc & 80.0 & 54.3 & 42.4 & 51.5 & 60.9 \\
\hline & $\mathrm{ppv}$ & 22.4 & 38.3 & 36.8 & 37.9 & 36.4 \\
\hline & npv & 87.9 & 84.8 & 65.6 & 76.7 & 84.7 \\
\hline
\end{tabular}

